\title{
Methods of gravitational wave detection in the VIRGO Interferometer
}

F. Acernese ${ }^{6}$, P. Amico ${ }^{10}$, M. Alshourbagy ${ }^{11}$, F. Antonucci ${ }^{12}$, S. Aoudia ${ }^{7}$, P. Astone ${ }^{12}$, S. Avino 6 , D. Babusci ${ }^{4}$, G. Ballardin ${ }^{2}$, F. Barone ${ }^{6}$,

L. Barsotti ${ }^{11}$, M. Barsuglia ${ }^{8}$, F. Beauville ${ }^{1}$, S. Bigotta ${ }^{11}$, S. Birindelli ${ }^{11}$, M.A. Bizouard ${ }^{8}$, C. Boccara ${ }^{9}$, F. Bondu ${ }^{7}$, L. Bosi $^{10}$, C. Bradaschia ${ }^{11}$, S. Braccini ${ }^{11}$,A. Brillet ${ }^{7}$, V. Brisson ${ }^{8}$, L. Brocco $^{12}$, D. Buskulic ${ }^{1}$, E. Calloni ${ }^{6}$, E. Campagna ${ }^{3}$, F. Carbognani ${ }^{2}$, F. Cavalier ${ }^{8}$, R. Cavalieri ${ }^{2}$, G. Cella ${ }^{11}$, E. Cesarini ${ }^{3}$, E. Chassande-Mottin ${ }^{7}$, N. Christensen ${ }^{2}$, C. Corda $^{11}$, A. Corsi ${ }^{12}$, F. Cottone ${ }^{10}$, A.-C. Clapson ${ }^{8}$, F. Cleva ${ }^{7}$,

J.-P. Coulon ${ }^{7}$, E. Cuoco ${ }^{2}$, A. Dari ${ }^{10}$, V. Dattilo ${ }^{2}$, M. Davier ${ }^{8}$, M. del Prete ${ }^{2}$, R. De Rosa ${ }^{6}$, L. Di Fiore ${ }^{6}$, A. Di Virgilio ${ }^{11}$, B. Dujardin ${ }^{7}$, A. Eleuteri ${ }^{6}$, I. Ferrante ${ }^{11}$, F. Fidecaro ${ }^{11}$, I. Fiori ${ }^{11}$, R. Flaminio ${ }^{1}, 2$, J.-D. Fournier ${ }^{7}$, O.Francois ${ }^{2}$, S. Frasca ${ }^{12}$, F. Frasconi ${ }^{2}, 11$, L. Gammaitoni ${ }^{10}$, F. Garufi ${ }^{6}$, E. Genin $^{2}$, A. Gennai ${ }^{11}$, A. Giazotto ${ }^{11}$, G. Giordano ${ }^{4}$, L. Giordano ${ }^{6}$,

R. Gouaty ${ }^{1}$, D. Grosjean ${ }^{1}$, G. Guidi ${ }^{3}$, S. Hebri ${ }^{2}$, H. Heitmann ${ }^{7}$, P. Hello ${ }^{8}$, S. Karkar ${ }^{1}$, S. Kreckelbergh ${ }^{8}$, P. La Penna ${ }^{2}$, M. Laval ${ }^{7}$, N. Leroy ${ }^{8}$, N. Letendre ${ }^{1}$, B. Lopez ${ }^{2}$, Lorenzini $^{3}$, V. Loriette ${ }^{9}$, G. Losurdo ${ }^{3}$, J.-M. Mackowski ${ }^{5}$, E. Majorana ${ }^{12}$, C. N. Man $^{7}$, M. Mantovani ${ }^{11}$, F. Marchesoni ${ }^{10}$, F. Marion ${ }^{1}$, J. Marque $^{2}$, F. Martelli ${ }^{3}$, A. Masserot ${ }^{1}$, M. Mazzoni ${ }^{3}$, L. Milano ${ }^{6}$, F. Menzinger ${ }^{2}$, C. Moins ${ }^{2}$, J. Moreau ${ }^{9}$, N. Morgado ${ }^{5}$, B. Mours ${ }^{1}$, F. Nocera ${ }^{2}$, A. Pai ${ }^{12}$, C. Palomba ${ }^{12}$,

F. Paoletti ${ }^{2}, 11$, S. Pardi ${ }^{6}$, A. Pasqualetti ${ }^{2}$, R. Passaquieti ${ }^{11}$, D. Passuello ${ }^{11}$, B. Perniola ${ }^{3}$, F. Piergiovanni ${ }^{3}$, L. Pinard ${ }^{5}$, R. Poggiani ${ }^{11}$, M. Punturo ${ }^{10}$, P. Puppo ${ }^{12}$, K. Qipiani ${ }^{6}$, P. Rapagnani ${ }^{12}$, V. Reita ${ }^{9}$, A. Remillieux ${ }^{5}$,

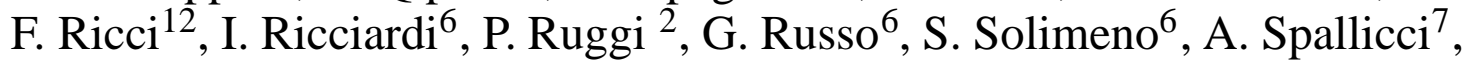
R. Stanga ${ }^{3}$, T. Marco ${ }^{11}$, M. Tonelli ${ }^{11}$, A. Toncelli ${ }^{11}$, E. Tournefier ${ }^{1}$, F. Travasso ${ }^{10}$, C. Tremola ${ }^{11}$, G. Vajente ${ }^{11}$, D. Verkindt ${ }^{1}$, F. Vetrano ${ }^{3}$,

A. Viceré ${ }^{3}$, J.-Y. Vinet ${ }^{7}$, H. Vocca ${ }^{10}$ and M. Yvert ${ }^{1}$

${ }^{1}$ Laboratoire d'Annecy-le-Vieux de Physique des Particules (LAPP), IN2P3/CNRS, Université de Savoie,Annecy-le-Vieux, France;

${ }^{2}$ European Gravitational Observatory (EGO), Cascina (Pi), Italia;

${ }^{3}$ INFN, Sezione di Firenze/Urbino, Sesto Fiorentino, and/or Università di Firenze, and/or Università di Urbino, Italia;

${ }^{4}$ INFN, Laboratori Nazionali di Frascati, Frascati (Rm), Italia; ${ }^{5}$ LMA, Villeurbanne, Lyon, France;

${ }^{6}$ INFN, sezione di Napoli and/or Università di Napoli "Federico II" Complesso Universitario di Monte S.Angelo, and/or Università di Salerno, Fisciano (Sa), Italia;

${ }^{7}$ Departement Artemis - Observatoire de la Côte d'Azur, BP 4220906304 Nice, Cedex 4, France; 
${ }^{8}$ Laboratoire de l'Accélérateur Linéaire (LAL), IN2P3/CNRS Université de Paris-Sud, Orsay, France;

${ }^{9}$ ESPCI, Paris, France;

${ }^{10}$ INFN, Sezione di Perugia and/or Università di Perugia, Perugia, Italia;

${ }^{11}$ INFN, Sezione di Pisa and/or Università di Pisa, Pisa, Italia;

${ }^{12}$ INFN, Sezione di Roma and/or Università "La Sapienza", Roma, Italia.

\begin{abstract}
The gravitational wave detector VIRGO is in the final commissioning phase and some preliminary data has already been acquired. The aim of VIRGO is to directly detect gravitational waves emitted by compact objects at the time of coalescence and by other astrophysical sources. Here we report on the present sensitivity for detecting these sources and what we will achieve in the near future. We also discuss some details of our data analysis methods and we show some preliminary results related to the fraction of the Universe that we can observe.
\end{abstract}

Keywords: <Enter Keywords here>

PACS: $04.80 . \mathrm{Nn}$, 95.55.Ym

\title{
INTRODUCTION
}

Einstein's Theory of General Relativity predicted the existence of gravitational waves (GW) in 1916. In the weak field approximation Einstein equation's can be linearized, admitting wave-like solutions, corresponding to weak, transverse waves, propagating in vacuum with the speed of light[1]. It is possible write a general GW solution, as the sum of two components $\mathbf{h}_{\mu v}=a h^{+}+b h^{\times}$, where $h^{+}$and $h^{\times}$are the two possible polarization state.

Up to now, GW have not been directly detected. But there are many indirect pieces of evidence that confirm the existence of these waves, most notably, the observation of the binary pulsar PSR 1913+16[2]; the Nobel Prize was awarded in 1993 for the discovery of this binary neutron star system. . This system loses orbital energy in perfect agreement with the theory of general relativity, which predicts energy loss via GW emission.

In order to demonstrate directly the existence of GW, we need an experimental apparatus that we call a "gravitational wave antenna". The gravitational field weakly interacts with the matter, and this is the main problem to address. Moreover, it seems impossible to create on Earth a source of such GW signals, strong enough to be detected by any realizable experiment. Therefore, we have to move our attention to very massive and dense systems with asymmetric mass distributions, moving at near-relativistic speeds. Only since the early 1990s have physicists believed that technology is at the point where direct detection of gravitational waves is possible ${ }^{1}$.

\section{GRAVITATIONAL WAVES AND INTERFEROMETRIC GW DETECTORS}

In considering gravitational waves effect on free masses, we start by examining figure 1 . We show an ideal circular mass distribution centered on a Michelson interferometer. A

${ }^{1}$ Important experiments had been conducted in the past, but without success[3] 


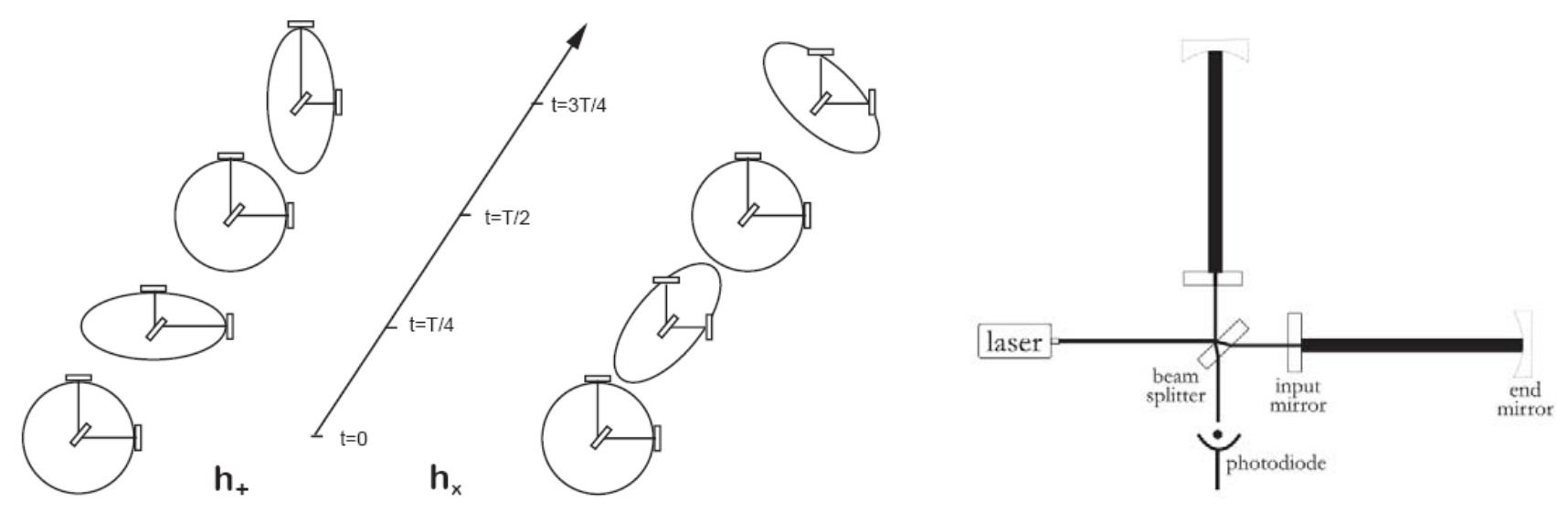

FIGURE 1. (LEFT)Effect of GW polarizations over a period. The figure shows the effect of a wave on a Michelson interferometer - (RIGHT)Michelson interferometer schema

picture of such device is reported on figure 1 on the right. It produces interference fringes by splitting a beam of monochromatic light, via the beam-splitter mirror. When the two beams strike the end-mirrors and are reflected back, an interference pattern results on the photodiode. For the our purpose the two end-mirrors are free-masses.

If a $\mathrm{GW}$ is acting on the system, coming from an orthogonal direction with respect to the plane containing the interferometer, it produces a deformation in such a way that the two arm lengths change asymmetrically. This is seen by the beams traveling between the mirrors as a change in the interference condition. In this way we can measure the displacement $\Delta L$ of the test masses by monitoring the interference pattern. Given the dimensionless GW amplitude $\mathbf{h}$, we can write: $h / 2 \approx \frac{\Delta L}{L}$. This implies that the longer the interferometer arms $L$ are, the better sensitivity we can reach. Hence, in this configuration the Michelson interferometer can be used as GW detector, fitting well the gravitational wave action on free masses.

\section{Virgo Detector}

VIRGO (http://www.virgo.infn.it) is a 3 kilometer interferometer built through a French-Italian collaboration, located in Cascina near Pisa, Italy (see figure 2)[4]. It has been funded by the French CNRS (Centre National de la Recherche Scientifique) and the Italian INFN (Istituto Nazionale di Fisica Nucleare). This collaboration involves 11 laboratories and more than 150 scientists. The main goal of the collaboration effort is $\mathrm{GW}$ observation at distances up to $15 \mathrm{Mpc}$, corresponding the Virgo cluster. From there binary inspiral events with a GW amplitude of $h \approx 10^{-21}-10^{-22} 1 / \sqrt{\mathrm{Hz}}$ can be expected.

The technologies implemented in this experiment permit to one realize one of the most promising GW detectors based on Michelson interferometer. It is capable of achieving displacement measurement accuracy at least of the order of $10^{-18} \mathrm{~m}$, maintaining the quasi-free masses condition for the end-mirrors and reducing any noise contamination as best as possible. The entire interferometer optics are extremely well isolated from the 


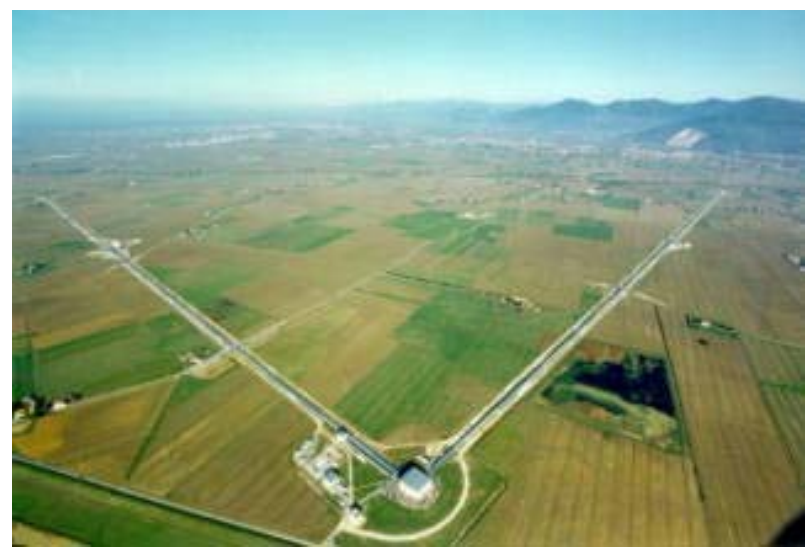

FIGURE 2. External view of the Virgo experiment. The two $3 \mathrm{~km}$ long arms and the central building are well visible.

environment, in order to be sensitive to GW. The Italian and French Virgo collaboration have developed advanced techniques with high reflectivity mirrors, mirror suspensions, high power ultra stable lasers, seismic isolation, and alignment control. Inside each interferometer arm is a Fabry-Perot cavity, in order to extend the optical length of each arm up to $120 \mathrm{~km}$.

We use a sensitivity curve to express the interferometer detector capability on measuring displacements at different frequencies. In figure 3 we show the expected theoretical Virgo sensitivity curve and noise contributions. Roughly speaking, we can say that given a gravitational wave signal in the frequency domain, we can extract and use the part of signal falling in the sensitivity curve with lower $h$ values. The Virgo detector has a wide response region, between $24-10000 \mathrm{~Hz}$.

We can divide noise sources effecting the interferometer sensitivity in two main groups: noise producing disturbances on the mirror position, and interferometer output signal fluctuations. These noise contributions are shown in figure 3 . We can highlight some of these: below $[<4 \mathrm{~Hz}]$ there is the residual seismic noise; between $[2-10] \mathrm{Hz}$ there is the noise induced by local gravity field fluctuations, called Newtonian noise; between $[10-30] \mathrm{Hz}$ there is the pendulum thermal noise, due to the mirrors' suspensions; between $[30-300] \mathrm{Hz}$ there is the mirror thermal noise; and above $[300] \mathrm{Hz}$ there is the photon shot noise.

\section{GRAVITATIONAL WAVE SOURCES}

Dynamically different sources emit gravitational waves with distinctive characteristics in the frequency domain. We describe the most important expected sources:

- inspiraling coalescing binary stars The signal emitted by a coalescing binary system is produced by two compact stars, orbiting around each other, in their evolving final phase as distinct objects. Analytical predictions [5] show that these are the most promising sources for GW emission, in particular if we are interested in neutron star/neutron star ( NS/NS) and black hole/black hole $(\mathrm{BH} / \mathrm{BH})$ populations. 


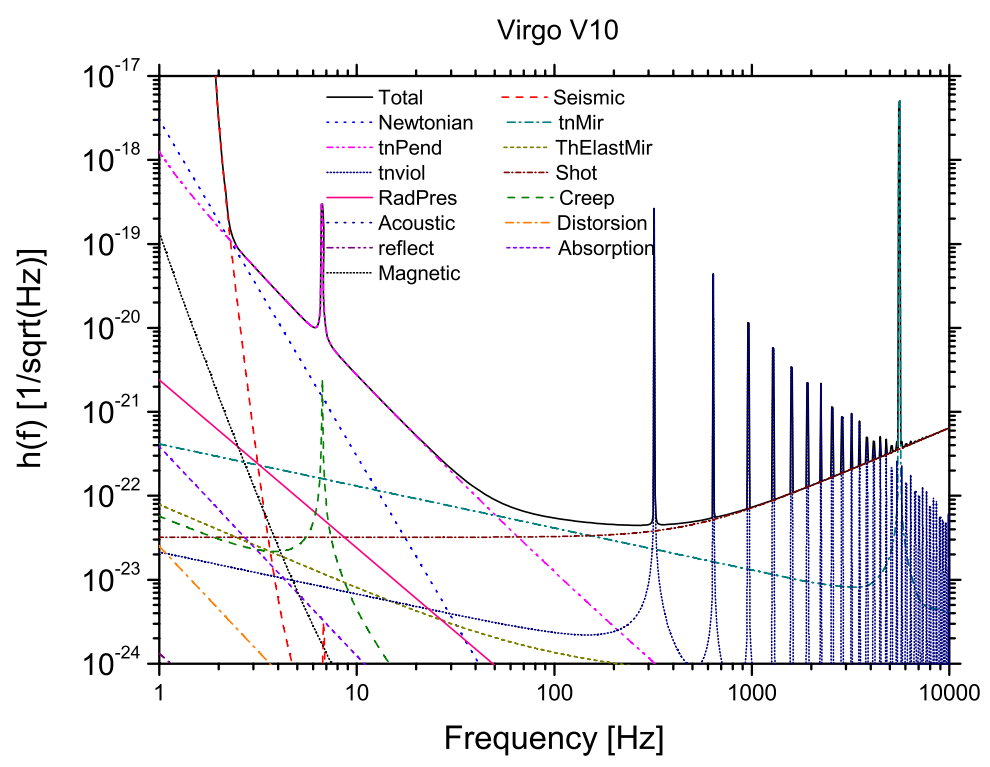

FIGURE 3. On the horizontal axis we show the frequencies, expressed in units of $H z$, and on the vertical axis we show the sensitivity, expressed with the dimensionless strain $h$, in units of $1 / \sqrt{(} \mathrm{Hz})$.

These types of signals are short and theoretically well modeled, characterized by an envelope in frequency and amplitude as the two objects coalesce. The detection algorithm used is the optimal filter, namely a matched filter[6].

- Burst signal This sporadic signal will be difficult to detect with the first generation of Michelson ground-based detectors. Supernovae core collapse, gamma-ray bursts (NS/NS, BH/NS, hypernovae) and dynamical instabilities in proto-neutron stars could produce these burst signals. These are short-lived and roughly modeled signals.

- Periodic signal are emitted by pulsars. Non asymmetric motion of neutron stars (which can be tracked over many cycles) will produce a periodic signal of gravitational waves. Interestingly, because these motion are so regular and long-lived, the resulting signal is nearly monochromatic. Doppler shifts complicate this analysis due to the earth's motion. The waves can be used to monitor known pulsars, while it is also possible to do searches on the sky to find unknown pulsars.

- Stochastic Signals Similar to the cosmic microwave background and the unprobed cosmic neutrino background, there exists a cosmic gravitational wave background.

\section{VIRGO GRAVITATIONAL WAVE DETECTION}

Since coalescing binaries are the most promising sources, we will consider the matched filter algorithm. It essentially compares (correlation) a set of theoretical coalescing binary $(\mathrm{CB})$ reference signals, called templates, with the signal output of the detector. 


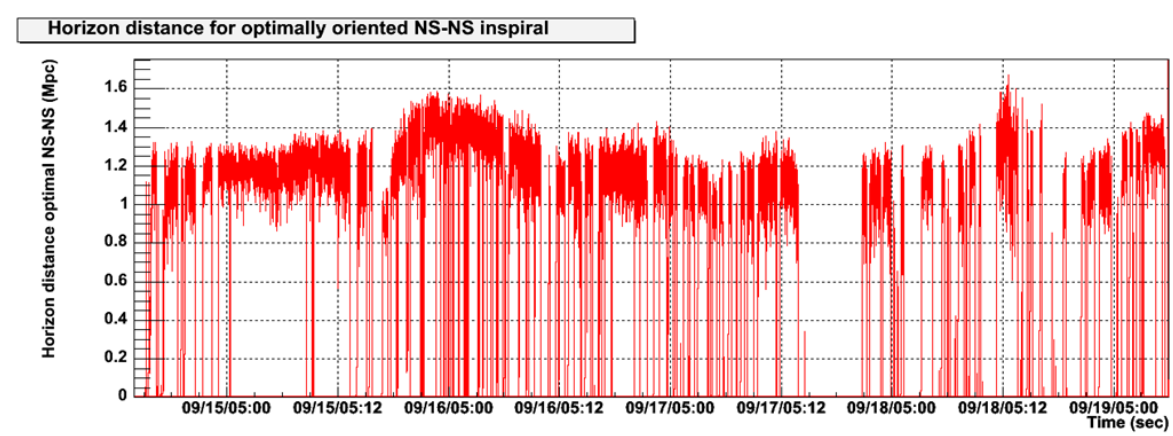

FIGURE 4. Horizon distance during the $\mathrm{C} 7$ commissioning run for an optimally oriented $1.41 .4 M_{s}$ NS/NS binary with SNR $=8$.

The result of this analysis provide is the probability that similar signals are present in the Virgo data set[7][8]. This information is proportional to the signal-to-noise ratio (SNR). When the CB signal is strongest, it appears cleaner in the matched filter output. In the analysis procedure we define a threshold SNR level, selecting only events above this value.

Detection probability can change respect to many parameters: GW source physical parameters, interferometer-source relative orientation, and interferometer sensitivity. If we look at a reference source signal having defined masses and a specific detector sensitivity, it is possible to estimate the maximum distance at which we can observe such event. We define this distance as the horizon distance (HD), usually expressed in $\mathrm{Mpc}$ and representing the far border at which we can make a detection.

Hence, a larger horizon distance corresponds to a better detector sensitivity and a bigger volume of explorable space. In addition, the detection rate probability increases because the number of "observable" sources is statistically higher. Usually for a reference signal, we use the NS/NS pair of $1.41 .4 M_{S}$ with an $S N R=8$ and optimally oriented with respect to the detector.

The Virgo collaboration has performed many data taking runs in the last few years, in order to measure and verify the interferometer status and improvements. The HD is one of the most important pieces of information about the detector status and performance.

In the 2005, Virgo had big improvements, leading from an average horizon distance of few $k p c$ to $1.2 M p c$. The highest value was reached during September 2005, when the collaboration had an important run, called C7 [9]. We show in figure 4 the detector performance during this data taking run, using the horizon distance as a measure. It is clear that the HD is not constant, because the Virgo sensitivity changes with time. The interferometer was able to cover the 110.78 hours with duty cycle:

- $70 \%$ locked

- $65 \%$ science mode

Virgo is now moving to the end of commissioning activities, and this will lead to it achieving its target sensitivity in 2007, for which we will have a HD of approximately $20 \mathrm{Mpc}$. For the first generation of interferometric detectors, theoretical predictions show a detection rate between $1 \mathrm{CB}$ event every 140 years to 1 event $\mathrm{CB}$ every 2 years. These 
predictions are based on population synthesis evolution models, and direct observations.

In order to achieve higher event rates, improvements to actual detectors have been planned, and these will be applied in the near future. There are also projects concerning next generation experiments, with significantly better sensitivity, implementing new technological solutions. In order to increase the detection rate, we need to move the horizon distance at least to the Great Attractor. In fact the future Virgo detector, called Virgo Advanced, has a design sensitivity that promises to reach such distances, 120$200 \mathrm{Mpc}$. In this situation, theoretical predictions provide event rates between 1 and 4000 (or more) events per year[10].

\section{CONCLUSIONS}

Presently the commissioning activities on the Virgo detector are in progress, thereby improving the HD. We hope to be close to the design sensitivity in the first half of 2007. In the same year the Virgo collaboration will start long data taking activities, in order to get and exchange simultaneous data with other analogous experiments (e.g. LIGO). This analysis, called network analysis, provides a significant increase in the detection probability [11]. There are other possible coincidence analysis, for example, with astrophysical observations (e.g. gamma ray burst); these events could be associated with a significant gravitational wave source.

Probably the next two years could be full of good news, with the possible first direct observation of gravitational waves.

\section{REFERENCES}

1. e.g. Misner, Charles, Thorne, Kip S. \& Wheeler, John Archibald (1973), "Gravitation", San Francisco: W. H. Freeman. ISBN 0-7167-0344-0.

2. J. M. Weisberg, J. H. Taylor, " Relativistic Binary Pulsar B1913+16: Thirty Years of Observations and Analysis " Binary Radio Pulsars ASP Conference Series, Vol. TBD, 2004 eds. F.A. Rasio \& I.H. Stairs

3. J. Weber, "Gravitational-Wave-Detector Events," Phys. Rev. Lett. 20, 1307 (1968).

4. F. Acernese et al. AIP Conf.Proc 794:307-310,2005 - Prepared for 17th Conference on High Energy Physics (IFAE 2005) (In Italian), Catania, Italy, 30 Mar - 2 Apr 2005

5. L. Blanchet, T. Damour, G Esposito-Farese, B. R. Iyer, "Gravitational radiation from inspiralling compact binaries completed at the third post-Newtonian order", Physical Review Letters, 93, 091101 (2004)

6. e.g. J. Owen, B. S. Sathyaprakash "Matched filtering of gravitational waves from inspiraling compact binaries: Computational cost and template placement" ??Phys.Rev. D60 (1999) 022002

7. P. Amico, L. Bosi, C. Cattuto, L. Gammaitoni, F. Marchesoni, M. Punturo, F. Travasso, H. Vocca, Comp.Phys.Comm. 153, 179 (2003).

8. F. Marion et al., (The Virgo Collaboration), Proceedings of the Rencontres de Moriond 2003, Gravitational Waves and Experimental Gravity (2004).

9. F Acernese et al, Class. Quantum Grav. 23 (2006) S635ÜS642

10. V.Kalogera et al., "The Cosmic Coalescence Rates for Double Neutron Star Binaries" Astrophys.J. 601 (2004) L179-L182

11. F. Beauville et al. "Benefits of joint LIGO - Virgo coincidence searches for burst and inspiral signals" J.Phys.Conf.Ser. 32 (2006) 212 